\title{
Effect of joint range of motion on health-related quality of life in children with hemophilia
}

\author{
Manal Anwar El Hawary ${ }^{a}$, Hanaa Hassan EL Dash ${ }^{a}$, Nermeen Ahmed Foaud ${ }^{b}$, \\ Mona Hussien Mohamed ${ }^{c}$
}

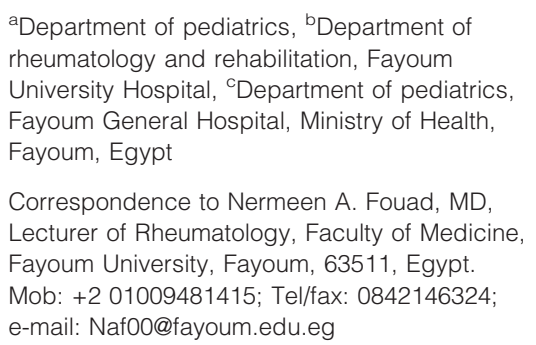

aDepartment of pediatrics, ${ }^{b}$ Department of rheumatology and rehabilitation, Fayoum

University Hospital, 'Department of pediatrics, Fayoum General Hospital, Ministry of Health,

Fayoum, Egypt

Correspondence to Nermeen A. Fouad, MD, Lecturer of Rheumatology, Faculty of Medicine, Fayoum University, Fayoum, 63511, Egypt. Mob: +2 01009481415; Tel/fax: 0842146324 e-mail: Naf00@fayoum.edu.eg

Received 18 February 2019 Accepted 17 April 2019

Egyptian Rheumatology \& Rehabilitation 2019, 46:237-243

\begin{abstract}
Background
Assessment of quality of life (QOL) in patients with hemophilia is important for the disease outcome. In patients with hemophilia, repeated occurrences of hemarthrosis lead to limitations in range of motion (ROM) of major joints.

Objective

The aim of this work is to assess joint ROM and detect the presence of limitation of joint ROM in patients with hemophilia and their effect on the QOL in these patients. Patients and methods

The study included 25 children with hemophilia recruited from Pediatric Hematology Unit, Fayoum University Hospital, during the period from June 2016 to December 2016. Their ages ranged between 4 and 16 years. Large joint examination and measurement of joint ROM were done at rheumatology clinic using goniometry by rheumatologist. QOL was assessed by using the hemophilia QOL questionnaire. Results

The most impaired dimensions of QOL were family, treatment, and physical domains. Total health-related quality of life (HRQOL) score, physical health, view of self, and sport and school scores were found worse with increasing age. Patients who had target joints had poor QOL score. Limitation of movement (LOM) of the knee and ankle joints mainly impaired the physical and social aspects and the overall HRQOL, and LOM of shoulder joint impaired the treatment domain.

\section{Conclusion}

Age of the patient was found a factor affecting the total HRQOL score, in addition to physical, view of self, and sport and school dimensions. LOM of the knee and ankle joints mainly impaired physical and social aspects and the overall HRQOL.
\end{abstract}

\section{Keywords:}

hemophilia, joint limitation, quality of life, range of motion

Egypt Rheumatol Rehabil 46:237-243

(C) 2019 Egyptian Society for Rheumatology and Rehabilitation

1110-161X

\section{Introduction}

Hemophilia is a bleeding disorder of $\mathrm{X}$-linked hereditary. Deficiency in coagulation factor VIII for hemophilia A and factor IX for hemophilia B is the cause of this disease [1]. Patients with severe hemophilia present with spontaneous bleeding since early childhood, chiefly in the musculoskeletal system (muscles and large joints) and, less commonly, mucosal or cerebral hemorrhages. Joint bleeding (hemarthrosis) results in synovial hypertrophy and damage of the cartilage, leading to joint destruction with recurrent bleeding episodes (hemophilic arthropathy) [2].

Overall, $85 \%$ of all bleeding events occur in joints in patients with severe hemophilia, with the ankle, knee, elbow, hip, and shoulders being the most commonly affected joints [3]. Recurrent happenings of hemarthrosis and synovitis with joint destruction and pain lead to limitations in joint range of motion (ROM). Joint ROM limitations in patients with hemophilia are increased with more advanced age, high BMI, and non-white ethnicity [4].
Quality of life (QOL) is defined - in correspondence to the WHO definition of health - as patient-perceived well-being and function in terms of physical, emotional, mental, social, and behavioral life domains [5]. Recently, the QOL of children having chronic diseases has received increasing consideration, mainly for frequent pediatric health conditions such as asthma or life-threatening conditions such as leukemia. Conversely, the QOL of young people with uncommon diseases like hemophilia has been largely ignored. It is essential to know more about QOL in this group of patients for better evaluation and improving the care these patients obtain [6]. Instruments for measuring QOL in hemophilia have been made only over the past few years. The hemophilia quality-of-life (Hemo-QOL) instrument is one of the first self-report tools, which is constructed as a set of disease-specific and

This is an open access journal, and articles are distributed under the terms of the Creative Commons Attribution-NonCommercial-ShareAlike 4.0 License, which allows others to remix, tweak, and build upon the work non-commercially, as long as appropriate credit is given and the new creations are licensed under the identical terms. 
age-related questionnaires to measure $\mathrm{QOL}$ in children and adolescents with hemophilia, and this instrument was established in the course of the Hemo-QOL project [7].

Chen et al. [8] hypothesized that ROM limitations in certain major joints of adult patients with hemophilia largely affect their health-related quality of life (HRQOL). However, the effect of ROM limitations of the major joints on HRQOL in children with hemophilia has been inadequately studied before. The aim of the present study is to assess joint function and detect the presence of limitation of joint ROM in patients with hemophilia and their effect on the QOL in these patients.

\section{Patients and methods}

This is a cross-sectional study that included 25 children with hemophilia A and B, their age ranged between 4 and 16 years. All included patients were subjected to full history taking with special emphasis on age of onset of the disease, frequency of attacks, type of therapy administered, and presence of complications. General examination was done to detect pallor, bleeding from sites other than joints, and manifestations of complications. Grading of severity of bleeding into slight, moderately severe, severe, and very severe by assessing of factor activity level was as follow: mild (5-40\% of normal activity), moderate ( $1-5 \%$ of normal activity), and severe ( $<1 \%$ of normal activity) $[9,10]$.

HRQOL of hemophilic patients was assessed by newly developed disease-specific Hemo-QOL. The HemoQOL is a self-report specific questionnaire with three versions for different age groups: group I included younger children with ages 4-7 years and their questionnaire included 21 items pertaining to eight dimensions (physical health, feelings, view, family, friends, others, sport and school/kindergarten, and treatment). Group II included school children aged 8-12 years and the self-administered questionnaire contains two additional domains (perceived support and dealing), with overall 64 items. Group III included adolescents 13-16 years of age and their questionnaire was expanded with further additional domain (future), consisting of 77 items.

The psychometric structure of the questionnaire has acceptable psychometric properties for the three age group versions [11]. For Hemo-QOL questionnaires, a total score summarizing HRQOL for the patient and domain scores is calculated. Scores range from 0 to 100 , with lower scores indicating better hemophilia-related
QOL [12]. High values indicate high impairments in HRQOL. Basically high score represents low QOL and scoring involves the following steps:

Assorting numbers to the response scale, which for the age groups II and III are 1=never, 2=seldom, $3=$ sometimes, $4=$ often, and $5=$ all the time. Please note, for the age group I, scoring is $1=$ never, $2=$ sometimes, and $3=$ very often.

Measurement of ROM of 17 movements of the five major joints (bilateral hips, knees, ankles, elbows, and shoulders) was performed at the Rheumatology Outpatient Clinic, Fayoum University Hospital, by a senior rheumatology physician using the goniometry technique. Large joints assessment on examination was performed for the presence of hemarthrosis and presence of target joints (three or more spontaneous bleeds into the joint within a consecutive 6-month period). The measuring method was based on the standard technique described in the guide $[13,14]$ (Table 1). A difference in angles between normal ROM and the participant's ROM of an individual movement was defined as deficit of ROM of the individual movement, and was obtained by subtracting the participant's ROM of the individual movement from normal ROM. Therefore, the deficit of ROM of individual movement was a positive value [8].

The study was approved through ethical committee, Fayoum University. Verbal informed assent or consent from all the study patients or their guardians respectively was taken before data collection.

Table 1 Normal angles of range of motion of major joints

\begin{tabular}{lcc}
\hline Joints & Action & Degrees of motion \\
\hline Shoulder & Flexion & $0-180$ \\
& Extension & $0-40$ \\
& Abduction & $0-180$ \\
& Internal rotation & $0-80$ \\
Elbow & External rotation & $0-90$ \\
& Flexion & $0-150$ \\
Hip & Extension & 0 \\
& Flexion & $0-100$ \\
& Extension & $0-30$ \\
& Abduction & $0-40$ \\
& Adduction & $0-20$ \\
& Internal rotation & $0-40$ \\
Knee & External rotation & $0-50$ \\
& Flexion & $0-150$ \\
Ankle & Extension & 0 \\
& Plantar flexion & $0-40$ \\
& Dorsiflexion & $0-20$ \\
\hline
\end{tabular}




\section{Statistical analysis}

Data were collected and coded to facilitate manipulation of the data and double entered into Microsoft Access, and data analysis was performed using SPSS software (Milton, QLD, Australia), version 18, in Windows 7. Simple descriptive analysis was done in the form of numbers and percentages for qualitative data, and arithmetic means as central tendency measurement, SDs as measure of dispersion for quantitative parametric data, and inferential statistic tests were as follows:

(1) For quantitative parametric data:

(a) In-depended Student's $t$ test used to compare measures of two independent groups of quantitative data.

(b) One-way analysis of variance test in comparing more than two independent groups of quantitative data.

(2) For qualitative data

(a) $\chi^{2}$ test to compare two of more than two qualitative groups.

The level $P$ value less than or equal to 0.05 was considered the cutoff value for significance.

\section{Results}

All patients in the study were males, with a mean age of $8.1 \pm 0.78$ years old and range between 4 and 16 years. Regarding mothers' education, 64\% were educated. Positive consanguinity was present in $16 \%$ of cases and $84 \%$ had a similar condition in the family.

Overall, 76\% experienced hemophilia A, and 24\% experienced hemophilia B. Moreover, 60\% had severe disease. Clinically hemarthrosis was present in $84 \%$ of our cases. All of our patients were on demand therapy, and only $32 \%$ of them received prophylactic treatment. The replacement therapy given was plasma in $88 \%$ of the patients, recombinant factor in $8 \%$, and one patient received both. Age of first bleeding episode was $7.4 \pm 1.8$ months, whereas age of first hemarthrosis was $3 \pm 0.22$ years, and ranged between 1 and 4 years.

Limitation in joint movement was present in $52 \%$ and mainly affecting the knees (76.9\%). Almost all cases belonging to the age group 13-16 years had target joints and limitation of movement (LOM). No association was found between hemarthrosis, LOM, target joint affection and disease severity, or types of replacement therapy.

There was no statistically significant difference $(P>0.05)$ between severity of hemophilia and QOL dimensions. Moreover, there was no significant difference in total QOL score of patients with hemarthrosis, LOM, and the type of treatment.

However, target joint affection was associated with a higher mean $\pm \mathrm{SD}$ QOL score $(P=0.04)$, indicating a poor QOL.

The treatment domain of QOL score was significantly higher in patients with LOM of the shoulder joint $(31.3 \pm 18)(P=0.04)$. Moreover, the future domain was significantly higher in patients with LOM of the knee joint $(60.9 \pm 4.7)(P=0.02)$. In addition, the physical activity $(82.5 \pm 4.1)(P=0.004)$, view of self $(73.3 \pm 6.5)$ $(P=0.001)$, other persons $(53.2 \pm 4.4)(P=0.03)$, sport and school domains $(81.7 \pm 5.1)(P=0.002)$, and total QOL $(56.9 \pm 3.5)(P=0.008)$ were higher in patients with LOM of the ankle joints.

The mean \pm SD) total QOL score of patients was 45.1 \pm 14.7 , ranging from 10.4 to 73.4 . The highest score was found for the family followed by the treatment and physical health domains $79.58 \pm 2.8,67.64 \pm 6$, and 66.86 \pm 3.9 , respectively. The lowest score observed was that of perceived support, with $30 \pm 7.8$ (Table 2).

Physical health, view of self, and sport and school scores were found worse with increasing age, where patients of the older age group had the highest scores (Table 3).

A positive correlation was found between the mean total QOL score and development of LOM in the elbow, knee, and ankle joints ( $P$ values of $0.01,0.03$, and 0.01 , respectively) (Table 4$)$.

Joint bleeding was analyzed, and a score was used according to the different questions of the HemoQOL questionnaire during the period of the past 4

Table 2 Description of different quality-of-life domains among the study group

\begin{tabular}{lccc}
\hline Hemo-QOL dimensions & Mean \pm SD & Minimum & Maximum \\
\hline Physical health score & $66.86 \pm 3.9$ & 25 & 96.43 \\
Feeling score & $40.71 \pm 4.2$ & 0 & 89.29 \\
View of self-score & $40.27 \pm 7.1$ & 0 & 94.44 \\
Family score & $79.58 \pm 2.8$ & 37.5 & 100 \\
Friend score & $45.50 \pm 7.1$ & 0 & 100 \\
Perceived support score & $30 \pm 7.8$ & 12.5 & 56.25 \\
Other persons score & $43 \pm 4.8$ & 0 & 75 \\
Sport and school score & $62.79 \pm 5.4$ & 0 & 100 \\
Dealing with hemophilia score & $38.57 \pm 4.8$ & 25 & 71.43 \\
Treatment score & $67.64 \pm 6$ & 0 & 100 \\
Future score & $52.5 \pm 9.2$ & 18.75 & 68.75 \\
Global health score & $54 \pm 1.9$ & 50 & 75 \\
Total QOL & $45.1 \pm 14.7$ & 10.4 & 73.4 \\
\hline
\end{tabular}

Hemo-QOL, hemophilia quality-of-life; QOL, quality of life. 
Table 3 Comparisons of different quality-of-life domains among different age groups

\begin{tabular}{|c|c|c|c|c|}
\hline \multirow[t]{2}{*}{ Hemo-QOL dimensions } & \multicolumn{3}{|c|}{ Age groups } & \multirow[t]{2}{*}{$P$ value } \\
\hline & $\begin{array}{c}\text { 4-7 years }(N=15) \\
\text { Mean } \pm S E\end{array}$ & $\begin{array}{c}8-12 \text { years }(N=5) \\
\text { Mean } \pm S E\end{array}$ & $\begin{array}{c}\text { 13-16 years }(N=5) \\
\text { Mean } \pm S E\end{array}$ & \\
\hline Physical health score & $58.3 \pm 4.7$ & $70.7 \pm 7.4$ & $88.6 \pm 2.1$ & 0.005 \\
\hline Feeling score & $35.6 \pm 5.6$ & $50 \pm 11.7$ & $46.9 \pm 2.9$ & 0.3 \\
\hline View of self-score & $18.3 \pm 6.7$ & $71.7 \pm 10.7$ & $74.7 \pm 6$ & $<0.001$ \\
\hline Family score & $80 \pm 4.4$ & $76 \pm 3.3$ & $81.9 \pm 4.1$ & 0.8 \\
\hline Friend score & $50 \pm 9.7$ & $58.8 \pm 16.1$ & $18.7 \pm 4.4$ & 0.2 \\
\hline Perceived support score & - & $46.3 \pm 5.8$ & $30 \pm 7.8$ & 0.1 \\
\hline Other persons score & $35 \pm 6.8$ & $56.7 \pm 8.2$ & $53.3 \pm 4$ & 0.1 \\
\hline Sport and school score & $50 \pm 6.7$ & $80.6 \pm 7.9$ & $83.3 \pm 5.9$ & 0.009 \\
\hline Dealing with hemophilia score & - & $46.4 \pm 8.4$ & $30.7 \pm 2.1$ & 0.1 \\
\hline Treatment score & $73.3 \pm 9.3$ & $65.7 \pm 5.4$ & $52.5 \pm 8.1$ & 0.4 \\
\hline Future score & - & - & $52.5 \pm 9.2$ & - \\
\hline Global health score & $50 \pm 0$ & $65 \pm 6.1$ & $55 \pm 5$ & 0.003 \\
\hline Total quality of life & $37.5 \pm 3.2$ & $57.3 \pm 5.6$ & $55.7 \pm 3.7$ & 0.003 \\
\hline
\end{tabular}

Hemo-QOL, hemophilia quality-of-life. Bold values are significant at $p<0.05$.

Table 4 Correlation between bleeding score, domains of quality of life with degree of limitation of movement among study group

\begin{tabular}{|c|c|c|c|c|c|c|c|c|c|c|c|c|}
\hline LOM & Bleeding & PS & FS & vos & FaS & FrS & PS & SS & $\mathrm{DH}$ & TS & FS & TQOL \\
\hline \multicolumn{13}{|l|}{ Shoulder } \\
\hline$r$ & 0.48 & 0.18 & -0.09 & 0.19 & -0.24 & -0.27 & 0.27 & 0.09 & -0.22 & -.38 & 0.31 & 0.07 \\
\hline$P$ value & 0.02 & 0.4 & 0.7 & 0.4 & 0.3 & 0.2 & 0.4 & 0.7 & 0.5 & 0.07 & 0.6 & 0.7 \\
\hline \multicolumn{13}{|l|}{ Elbow } \\
\hline$r$ & 0.63 & 0.15 & 0.38 & 0.53 & -0.02 & -0.05 & 0.19 & 0.35 & 0.18 & -0.08 & 0.75 & 0.49 \\
\hline$P$ value & 0.002 & 0.02 & 0.06 & 0.007 & 0.9 & 0.8 & 0.6 & 0.08 & 0.6 & 0.7 & 0.1 & 0.01 \\
\hline \multicolumn{13}{|l|}{ Hip } \\
\hline$r$ & 0.34 & 0.16 & 0.03 & 0.14 & -0.12 & -0.08 & 0.38 & 0.16 & -0.22 & -0.25 & -0.07 & 0.10 \\
\hline$P$ value & 0.1 & 0.4 & 0.9 & 0.5 & 0.6 & 0.7 & 0.3 & 0.5 & 0.5 & 0.2 & 0.9 & 0.6 \\
\hline \multicolumn{13}{|l|}{ Knee } \\
\hline$r$ & 0.62 & 0.49 & 0.18 & 0.47 & 0.01 & -0.23 & -0.16 & 0.36 & 0.07 & 0.006 & 0.81 & 0.42 \\
\hline$P$ value & 0.002 & 0.01 & 0.4 & 0.01 & 0.9 & 0.3 & 0.7 & 0.07 & 0.8 & 0.9 & 0.1 & 0.03 \\
\hline \multicolumn{13}{|l|}{ Ankle } \\
\hline$r$ & 0.80 & 0.52 & 0.1 & 0.59 & -0.04 & -0.18 & 0.17 & 0.49 & -0.07 & -0.22 & 0.30 & 0.50 \\
\hline$P$ value & 0.001 & 0.007 & 0.6 & 0.002 & 0.9 & 0.4 & 0.6 & 0.01 & 0.8 & 0.3 & 0.6 & 0.01 \\
\hline
\end{tabular}

$\mathrm{DH}$, dealing with hemophilia score; FaS, family score; FrS, friend score; FS, feeling score; FS, future score; LOM, limitation of movement; OP, other persons score; PS, perceived support score; PS, physical health score; SS, sport and school score; TQOL, total quality of life score; TS, treatment score; VOS, view of self-score. Bold values are significant at $p<0.05$.

weeks before the study. Regarding the frequency of bleeding events, it was found that three patients did not bleed, 10 patients were exposed to bleeding more than two times. Experiencing bleeding attacks was found sometimes in 13 patients. Regarding the severity, eight patients had slight bleeding, five patients had moderately severe bleeding, 10 patients had severe bleeding, and two patients had very severe bleeding attacks. As for the strange feeling in joints, we found eight patients could not predict the bleeding before it occurred, nine patients rarely could get that feeling, and four always could predict the bleeding before it happened. According to staying quiet in bed, it was found that 13 patients seldom lied quiet in bed during the bleeding attack.
The mean bleeding score was $50.86 \pm 4.8$ and was significantly higher with increasing age $(79.04 \pm 3.6)$ $(P<0.001)$. The bleeding scores of shoulder, elbow, knee, and ankle joints were $74.6 \pm 11.1,72.1 \pm 5,67.1$ \pm 5.3 , and $74.6 \pm 3.1$, respectively.

Besides joint bleeding, all cases had history of bleeding from other sites: $40 \%$ during circumcision, $76 \%$ had epistaxis and bleeding gums, 8\% bleeding from injection sites, $8 \%$ hematemesis, $4 \%$ for hemoptysis, $76 \%$ had ecchymosis, intracranial hemorrhage in $12 \%$, hematuria in $12 \%$, and $4 \%$ after dental procedure. When comparing the different sites of bleeding among different age groups, mucosal bleeding and ecchymosis were more prevalent among cases of 
younger age (4-7 years) than other age groups, with a statistically significant difference $(P<0.05)$.

A positive correlation was found between the bleeding score and LOM of shoulder, elbow, knee, and ankle joints $(P=0.02,0.002,0.002$, and 0.001 , respectively), indicating that a higher score was associated with worsening of the LOM of the joints affected (Table 4).

There was a statistically significant positive correlation, with $P$ value less than 0.05 , between LOM of elbow joint score and physical and view domains, between knee joint score and physical and view domains, and between ankle joint score and physical, view, and sport domains, which indicates that higher LOM score is associated with an increase in the domains' score (Table 4).

\section{Discussion}

Joint disease in hemophilia resembles arthritis as the damage of joint, tendons, ligaments, and soft tissue is getting worse with time, resulting in pain, restricted movement, muscle atrophy, and reduced ROM [3].

Hemarthrosis has been found to be the most common (84\%) presenting feature in this study followed by mucosal bleeding and skin bleeds (76\%). Karim et al. [15], reported that hemarthrosis (82\%) and skin bleeds (72\%) were the most common clinical manifestations. Target joints were present in $36 \%$ of the studied hemophilic patients. The knee joint was the predominant target joint in $90 \%$ of cases and the ankle joint in 60\%. Moreover, Payal et al. [16], reported that $37.5 \%$ of hemophilic patients developed target joint, and the knee joint was the principal target joint in $28.57 \%$ of cases and ankle joint in $8.92 \%$.

In the present study, it was found that the first hemarthrosis was at the age of $3 \pm 0.22$ years and ranged between 1 and 4 years. This was in agreement with Abdel Ghany et al. [17], who found that the mean age of first hemarthrosis in severe hemophilic patients was $2.22 \pm 1$ years and ranged from 0.5 to 4.0 years.

LOM was present among $52 \%$ of patients; the most commonly affected joints were the knee in $76.9 \%$ followed by the ankle in $69.2 \%$. It was found that the target joints and LOM were higher among older age groups mainly in group III (age 13-16 years) with significant difference. As joint damage becomes progressive, the symptoms get worse with increasing age of patients with hemophilia. Moreover, another study reported that functional joint impairments were detected in nearly only one-tenth of European patients with a significant increase in the group III (age 13-16 years) [11].

However, Tantawy and colleagues, reported no differences between the three age groups regarding hemarthrosis and LOM. In addition, they stated that in the majority of the young age group (age 4-7 years) and in all patients with older age (age 13-16 years) at least one target joint was present [18]. Bullinger and Von Mackensen [5] reported that target joint and limitation were present in only half of the children: $61 \%$ of the old age group (age, 13-16 years) in contrast to young age group (age, 4-7 years).

The present work revealed that there was no significant association between hemarthrosis, target joints, and limitation of joints movement and different degrees of disease severity. However, another reported that the risk of developing a target joint was more in patients with severe hemophilia compared with those with moderate or mild hemophilia (33.1 vs. $18.8 \%$ and $5 \%$, respectively) [19].

Moreover, Prejs and colleagues, reported that the initial hemarthrosis occurs during the first year of life in patients with severe hemophilia, and $90 \%$ of youths with severe factor VIII or factor IX deficiency had at minimum one joint hemorrhage before the age of 4.5 years and more were exposed to develop hemophilic arthropathy with limitation of joint movement more than the other degrees of hemophilia [20].

Recently, HRQOL for different diseases had received increasing concern, but factors that may positively or negatively affect the QOL of children with hemophilia and the differences in these factors among different countries were not studied on a large scale [21].

The findings of the present study revealed that the mean total QOL score was $45.1 \pm 14.7$, ranging from 10.4 to 73.4. The most impaired dimensions of QOL were family, treatment, and physical domains and less impairment in support, dealing, and view of selfdomains. Physical health, view of self, and sport and school scores were found worse with increasing age ( $P=0.005,0.001$, and 0.009 , respectively), which indicates a poor QOL with increasing age. This is in agreement with that described in a study in Italy by Scalone et al. [22], in which the overall QOL was found to be negatively associated with age in both 
questionnaires. In addition, this is in agreement with a previous study in Egypt by Tantawy et al. [18], in which HRQOL was assessed by Hemo-QOL questionnaire. However, it is in contrast to a study in Iraq by Taha and Hassan [6] in which HRQOL was not affected by age of the patients.

Young children are mainly impaired in family and treatment dimensions, whereas older children have impairment in physical, sport, view of self, family, feeling, friend, and future scores. This is in agreement with that reported by Gringeri et al. [11] and Remor et al. [23]. This can be explained by the overprotection of parents for their young children, reflected in the family dimension, whereas older children had higher impairments in the social and physical dimension.

The present study showed that patients with target joints had a poor QOL compared with those without $(P=0.04)$. Moreover, patients with hemarthrosis and LOM had a poor QOL score compared with those without, but this did not reach significance. Karin and Berntop [24] stated that patients' QOL was severely affected hemarthrosis, recurrent joint bleeding, and LOM.

There was no significant difference in total QOL score regarding prophylactic treatment; this could be explained by small number of prophylactic treatment patients in the study and short time of study to show the effect of regular prophylaxis on QOL. However, Royal et al. [25], suggested that hemophilic patients who were treated prophylactically have a better QOL when compared with those who were treated on demand.

Results of the present study revealed that LOM of the knee and ankle joints mainly affected physical and social aspects and the overall HRQOL. The knee LOM made a significant effect on the future dimension; the ankle LOM had a significant effect on physical health, sport and school, view of self, other persons dimension, and the total QOL score; and shoulder LOM affected the treatment dimension. None of our patients had hip joints LOM. Chen et al. [8], in his study group (mean age, 36.9 years) showed that the deficit in ROM of the major joints in adults lead to diminished HRQOL in physical, mental, and social aspects, where limitation of the hip and ankle joints mainly affected the physical health dimension, whereas limitation of elbow and shoulder joints chiefly affect emotional and mental health.

\section{Conclusion}

Hemophilia like any other chronic disease affects different aspects of patients' life and also causes variable degrees of disability. Age of the patient was found as a factor affecting the total HRQOL score, in addition to physical, view of self, and sport and school dimensions, which indicated more affection of QOL with increasing age. LOM of the knee and ankle joints mainly impaired physical and social aspects and the overall HRQOL.

The limitations of this study is the small sample size and that most patients were on demand therapy.

\section{Financial support and sponsorship Nil.}

\section{Conflicts of interest}

There are no conflicts of interest.

\section{References}

1 Franchini M, Mannucci PM. Past, present and future of hemophilia: a narrative review. Orphanet $\mathrm{J}$ Rare Dis 2012; 7:24.

2 Lobet S, Hermans C, Lambert C. Optimal management of hemophilic arthropathy and hematomas. J Blood Med 2014; 5:207-218.

3 Roosendaal G, Lafeber FP. Blood induced joint damage in hemophilia. Semin Thromb Hemost 2003; 29:37-42.

4 Soucie JM, Cianfrini C, Janco RL, Kulkarni R, Hambleton J, Evatt B, et al. Joint range of motion limitations among young males with hemophilia. Blood 2004; 103:2467-2473.

5 Bullinger M, Von Mackensen S. Quality of life assessment in hemophilia. Hemophilia 2004; 10:26-33.

6 Taha M, Hassan M. Health related quality of life in children and adolescents with hemophilia in Basra,Southern Iraq. J Pediatr Hematol Oncol 2014; 36:179-184.

7 Pollak E, Mu Hlan H, Von Mackensen S, Bullinger M. The Haemo Qol Index: developing a short measure for health related quality of assessment in children and adolescents with hemophilia. Hemophilia 2006; 12:384-392.

8 Chen CM, Huang KC, Chen CC, Huang SU, Huang CE, Chen YY, et al. The impact of joint range of motion limitations on health-related quality of life in patients with haemophilia A. Haemophilia 2015; 21:176-184.

9 Paul-Scott J, Robert R. Montgomery hereditary clotting factor deficiencies (bleeding disorders). In Kliegman RM, Behrman RE, Jenson HB, Stanton $\mathrm{BF}$, eds. Nelson textbook of pediatrics. 19th ed. Philadelphia, PA: Saunders Elsevier; 2011. 470:1699-1701.

10 White GC II, Rosendaal F, Aledort LM, Lusher JM, Rothschild C, Ingerslev $\mathrm{J}$, Factor VIII and Factor IX Subcommittee. Definitions in hemophilia. Recommendation of the scientific subcommittee on factor VIII and factor IX of the scientific and standardization committee of the International Society on Thrombosis and Haemostasis. Thromb Haemost 2001; 85:560.

11 Gringeri A, Von Mackensen S, Auerswald G, Bullinger M, Garrido R, Kllermanne $\mathrm{E}$, et al. For the haemo-qol study, health status and healthrelated quality of life of children with haemophilia from six West European countries. Haemophilia 2004; 10:26-33.

12 Von Mackensen S, Bullinger M, and the Haemo-QoL Group. Development and testing of an instrument to assess the quality of life of children with haemophilia in Europe (Haemo-QoL). Haemophilia 2004; 10:17-25.

13 Norkin CC, White DJ. Measurement of joint motion: a guide to goniometry. 4th ed. Philadelphia, PA: F.A. Davis; 2009.

14 Cocchiarella L, Andersson GBJ. American Medical Association: guide to the evaluation of permanent impairment. 5th ed. Chicago, IL: AMA; 2001. 
15 Karim MA, Siddique R, Jamal CY, Islam A. Clinical profile of haemophilia in children in a tertiary care hospital. Bangladesh J Child Health 2013; 37:90-96.

16 Payal V, Sharma P, Janu Y. Joint health status of hemophilia patients in Jodhpur region. Indian J Hematol Blood Transfus 2015; 31:362-366.

17 Abdel Ghany HM, Hassab HM, El-Noueam KI. Hemophilic arthropathy: clinical, radiologic, and functional evaluation: a single-center experience in a limited resource country. Egypt Rheumatol Rehabil 2016; 43:35-40.

18 Tantawy A, Mackensen S, El-Laboudy M, Labib JH, Moftah F, El-Telbany MA, et al. Health-related quality of life in Egyptian children and adolescents with hemophilia A. Pediatr Hematol Oncol 2011; 28:222-229.

19 Alhaosawi MM. Target joint 'new concept of identification'. J Appl Hematol 2015; 6:35-38.

20 Prejs R, deKleijn P, Grobbee DE, vandenBerg M. The effects of postponing prophylactic treatment on long-term outcome in patient with severe hemophilia. Blood 2002; 99:2337-2341.
21 Bullinger M, von Mackensen S. Psycho-social determinants of quality of life in children and adolescents with hemophilia a cross cultural approach. Clini Psychol Psychother 2008; 15:164-172.

22 Scalone L, Mantovani LG, Mannucci PM, Gringeri A; COCIS Study Investigators. Quality of life is associated to the orthopedic status in haemophilic patients with inhibitors. Haemophilia 2006; 12:154-162.

23 Remor E, Young NL, Von Mackensen S, Lopatina EG. Disease specific quality of life measurement tools for hemophilia patients. Hemophilia 2004; 10:30-40.

24 Karin K, Berntorp E. Haemophilia and joint disease: pathophysiology, evaluation and management. J Comorb 2011; 1:51-59.

25 Royal S, Schramm E, Berntorp P, Giangrande M. Quality-of-life differences between prophylactic and on-demand factor replacement therapy in European haemophilia patients. Haemophilia 2002; 8:44-50. 